\title{
Synergistic activity of azoles with amiodarone against clinically resistant Candida albicans tested by chequerboard and time-kill methods
}

\author{
Correspondence \\ Shujuan Sun \\ sunshujuan888@163.com
}

Received 25 September 2007

Accepted 4 December 2007

\section{Qiongjie Guo, ${ }^{1}$ Shujuan Sun, ${ }^{2}$ Jinlong $\mathrm{Yu}^{1,3}{ }^{1,3} \mathrm{Yan} \mathrm{Li}{ }^{2}$ and Lili Cao ${ }^{4}$}

\author{
${ }^{1}$ School of Pharmaceutical Sciences, Shandong University, Jinan 250012, PR China \\ ${ }^{2}$ Department of Pharmacy, Shandong Provincial Qianfoshan Hospital, Jinan 250014, PR China \\ ${ }^{3}$ Department of Pharmacy, The Second Hospital of Shandong University, Jinan 250012, PR China \\ ${ }^{4}$ The General Surgical Central Laboratory, Shandong Provincial Qianfoshan Hospital, Jinan \\ 250014, PR China
}

\begin{abstract}
Candida albicans is the most common candidal pathogen, causing serious systemic disease in immunocompromised patients. Azoles are widely applied and largely effective; however, they are generally fungistatic and clinically resistant isolates are emerging increasingly. The present study provided in vitro evidence using a chequerboard technique that amiodarone is strongly synergistic with azoles against resistant C. albicans, with mean fractional inhibitory concentration indices of 0.01 and high-percentage synergistic interactions of $1250 \%$. A time-kill study performed by both colony counting and a colorimetric reduction assay confirmed the synergistic interaction, with $a \geqslant 2 \log _{10}$ decrease in c.f.u. $\mathrm{ml}^{-1}$ compared with the corresponding azoles alone. These results suggest the possibility of supplementing azoles with amiodarone to treat resistant C. albicans infections.
\end{abstract}

\section{INTRODUCTION}

Candida albicans is the predominant cause of virtually all types of candidiasis (Filler \& Sheppard, 2006). Coinciding with the occurrence of azole-resistant isolates, much effort has been undertaken to solve this by: (i) searching for new antifungal agents, such as posaconazole and ravuconazole, although new drugs for the treatment of these resistant infections are unlikely to appear soon enough and in sufficient numbers (Andes et al., 2006); (ii) developing new formulations of established agents, such as itraconazole (ITC), an intravenous formulation of which has overcome the poor and erratic bioavailability of the oral capsule formulation (Pfaller et al., 2005); and (iii) improving the efficacy of antifungal agents by using combination therapy, for example combining agents with different antifungal mechanisms (Barchiesi et al., 1998; Ghannoum et al., 1995; Girmenia et al., 2003) or combining antifungal and nonantifungal agents (Cruz et al., 2002; Quan et al., 2006).

Recently, the anti-arrhythmic drug amiodarone (AMD) was reported to have potent fungicidal activity against a broad range of fungi (Cryptococcus, Saccharomyces,

\footnotetext{
Abbreviations: AMD, amiodarone; $\mathrm{Bl}$, Bliss independence; $\mathrm{Cl}$, confidence interval; FIC, fractional inhibitory concentration; FICl, FIC index; FLC, fluconazole; ITC, itraconazole; LA, Loewe additivity; VRC, voriconazole; XTT, 2,3-bis(2-methoxy-4-nitro-5-sulfophenyl)-5-[(phenylamino)carbonyl]-2H-tetrazolium hydroxide.
}

Aspergillus, Candida and Fusarium) by testing the doubling times or cell weight (Courchesne, 2002). Researchers also showed that low doses of AMD acted synergistically with fluconazole (FLC) against C. albicans SC5314 by detecting the post-antifungal effect (Gupta et al., 2003). These results made us interested in a more detailed study of the in vitro activity of AMD in combination with azoles [FLC, ITC and voriconazole (VRC)] by conventional methods. In this paper, we present the combined effects of the three azoles and AMD against resistant $C$. albicans by multiple methods of testing and assessing. The strains used were new clinical isolates rather than standard C. albicans (Gupta et al., 2003).

\section{METHODS}

Strains. New clinical isolates of C. albicans strains were used in this study, namely CA10, CA15, CA16, CA135, CA137, CA5, CA8, CA12, CA14 and CA129. All strains were confirmed using standard mycological methods (Baumgartner et al., 1996; Fricker-Hidalgo et al., 1996; Pfaller et al., 1996) in the Microbiological Research Laboratory, Center of Health Research and Epidemic Prevention, Shandong Province, PR China. Candida parapsilosis ATCC 22019 was used as a quality-control strain. Isolates were stored at $-70{ }^{\circ} \mathrm{C}$, maintained at $4{ }^{\circ} \mathrm{C}$ and subcultured twice at $35{ }^{\circ} \mathrm{C}$ on Sabouraud dextrose agar.

Drugs. Standard powders of FLC (Shandong Chengchuang Pharmaceutical Technology Development), ITC (Xian-Janssen 
Pharmaceutical), VRC (Chengdu Qihe Pharmaceutical) and AMD hydrochloride (Zhejiang Excel Pharmaceutical) were used in the study. FLC was dissolved in sterile water at a final concentration of $2.56 \mathrm{mg} \mathrm{ml}^{-1}$. ITC, VRC and AMD were each dissolved in DMSO at a final concentration of $25.6 \mathrm{mg} \mathrm{ml}^{-1}$.

Medium. The medium was liquid RPMI 1640 (with L-glutamine, without sodium bicarbonate; Sigma) supplemented with glucose to a final concentration of $2 \%$ (Espinel-Ingroff et al., 2005a) and $0.165 \mathrm{M}$ MOPS (Sigma). The $\mathrm{pH}$ of the medium was adjusted to $7.0 \pm 0.1$ at $22{ }^{\circ} \mathrm{C}$ with $0.1 \mathrm{M} \mathrm{NaOH}$.

Drug susceptibility testing. MICs were tested according to the method of the Clinical and Laboratory Standards Institute (formerly the National Committee for Clinical Laboratory Standards) (CLSI, 2002). The inoculum was adjusted to achieve a final concentration of $0.5 \times 10^{3}$ to $2.5 \times 10^{3}$ c.f.u. $\mathrm{ml}^{-1}$. Drug dilutions were prepared in RPMI 1640 and the appropriate range of drug concentrations was chosen by an exploratory study. DMSO was always $\leqslant 1 \%$ of the total volume of medium. Plates were incubated at $35{ }^{\circ} \mathrm{C}$ for $48 \mathrm{~h}$. The $\mathrm{OD}_{492}$ was measured using a plate reader and background optical densities were subtracted from that of each well. Each isolate was tested in triplicate. MIC was defined as the lowest drug concentration showing $80 \%$ (or more) growth reduction compared with the drugfree control. Interpretive breakpoints for FLC [susceptible $(\mathrm{S}) \leqslant 8 \mu \mathrm{g}$ $\mathrm{ml}^{-1}$; susceptible-dose-dependent (SDD) $16-32 \mu \mathrm{g} \mathrm{ml}^{-1}$; resistant (R) $\left.\geqslant 64 \mu \mathrm{g} \mathrm{ml}^{-1}\right]$, ITC $\left(\mathrm{S} \leqslant 0.12 \mu \mathrm{g} \mathrm{ml}^{-1}\right.$; SDD $0.25-0.5 \mu \mathrm{g} \mathrm{ml}^{-1}$; R $\left.\geqslant 1 \mu \mathrm{g} \mathrm{ml}^{-1}\right)$ and VRC $\left(\mathrm{S} \leqslant 1 \mu \mathrm{g} \mathrm{ml}^{-1} ; \mathrm{SDD} 2 \mu \mathrm{g} \mathrm{ml}^{-1} ; \mathrm{R} \geqslant 4 \mu \mathrm{g}\right.$ $\mathrm{ml}^{-1}$ ) were used (Espinel-Ingroff et al., 2005b).

Drug interactions. Assays were performed in 96-well flat-bottomed microtitre plates (Costar) using a chequerboard method. The inoculum was used at a final concentration of $0.5 \times 10^{3}$ to $2.5 \times 10^{3}$ c.f.u. $\mathrm{ml}^{-1}$ and was verified by colony counting. The final concentrations of the drugs ranged from 0.125 to $128 \mu \mathrm{g} \mathrm{ml}^{-1}$ for FLC, 0.008 to $8 \mu \mathrm{g} \mathrm{ml}^{-1}$ for ITC and VRC, and 0.125 to $8 \mu \mathrm{g} \mathrm{ml}^{-1}$ for AMD. Each isolate was tested three times on different days. In order to assess the nature and intensity of the in vitro interactions between the three azoles and AMD, the data obtained were analysed using models based on two non-interaction theories: the Loewe additivity (LA) and the Bliss independence (BI) theories.

The non-parametric approach of LA is based on the fractional inhibitory concentration (FIC) index (FICI) model expressed as: $\Sigma \mathrm{FIC}=\mathrm{FIC}_{\mathrm{A}}+\mathrm{FIC}_{\mathrm{B}}=(\mathrm{MIC}$ of drug $\mathrm{A}$ in combination $/ \mathrm{MIC}$ of drug $\mathrm{A}$ alone $)+($ MIC of drug B in combination/MIC of drug B alone) (Te Dorsthorst et al., 2004). Among all of the $\Sigma$ FIC values calculated for each dataset, the FICI was determined as $\Sigma \mathrm{FIC}_{\text {min }}$ (the lowest $\Sigma \mathrm{FIC}$ ) when $\Sigma F C_{\max }$ (the highest $\Sigma F I C$ ) was less than 4; otherwise, the FICI was determined as $\Sigma \mathrm{FIC}_{\max }$ (Meletiadis et al., 2003). Synergy and antagonism were defined by FICIs of $\leqslant 0.5$ and $>4$, respectively. An FICI result of $>0.5$ but $\leqslant 4$ was considered indifferent (Odds, 2003).

The non-parametric approach of BI is based on the Prichard model defined as $\mathrm{E}_{i}=\mathrm{E}_{\mathrm{A}} \times \mathrm{E}_{\mathrm{B}}$, where $\mathrm{E}_{\mathrm{i}}$ is the predicted percentage of growth of the theoretical combination of drugs $\mathrm{A}$ and $\mathrm{B}$, respectively, and $\mathrm{E}_{\mathrm{A}}$ and $E_{B}$ are the experimental percentages of growth of each drug acting alone, respectively. Interaction is described by the difference $(\Delta \mathrm{E})$ between the predicted and measured percentages of growth at various concentrations. Because of the nature of the interaction testing with microtitre plates and a twofold dilution of either drug, this results in a $\Delta \mathrm{E}$ for each drug combination. When the mean $\Delta \mathrm{E}$ was positive and its $95 \%$ confidence interval (CI) among the replicates did not include 0 , statistically significant (SS) synergy was claimed; when the $\Delta \mathrm{E}$ was negative and its $95 \%$ CI did not include 0, SS antagonism was claimed. In any other case, BI was concluded. The $\Delta \mathrm{E}$ values obtained for each combination can be depicted on the $z$-axis to construct a 3D surface plot. Peaks above and below the 0 plane indicate synergistic and antagonistic combinations, respectively, whilst the 0 plane indicates the absence of SS interaction. As the plot only shows the interactions for each separate combination of the concentrations, a value is needed to summarize the interaction surface. This was achieved by calculating the sum percentage of all SS synergistic $(\Sigma \mathrm{SYN})$ and antagonistic ( $\Sigma \mathrm{ANT})$ interactions. Interactions with $<100 \%$ of SS interactions were considered weak, those with 100 $200 \%$ of SS interactions were considered moderate, and those with $>200 \%$ of SS interactions were considered strong. In addition, the numbers of SS synergistic and antagonistic combinations among the 77 combinations tested were calculated for each strain (Afeltra et al., 2004).

Time-kill assay. We investigated the activity of AMD with or without azoles against one resistant strain (CA10) at a starting inoculum of $10^{5}$ c.f.u. $\mathrm{ml}^{-1}$. A set of solutions was prepared: a growth control (drug-free), AMD alone $\left(4 \mu \mathrm{g} \mathrm{ml}^{-1}\right)$, azoles alone $\left(8 \mu \mathrm{g} \mathrm{ml} \mathrm{g}^{-1}\right.$ for FLC, $0.5 \mu \mathrm{g} \mathrm{ml}^{-1}$ for ITC and VRC) and combinations of AMD with each azole. All solutions were incubated at $35{ }^{\circ} \mathrm{C}$ without agitation (Klepser et al., 1998). At predetermined time points (0, 6, 12,24 and $48 \mathrm{~h}$ ), a $100 \mu \mathrm{l}$ sample was removed from each solution and serially diluted. A $100 \mu \mathrm{l}$ aliquot from each dilution was then streaked onto a Sabouraud dextrose agar plate to perform colony counting after incubation for $48 \mathrm{~h}$. A colorimetric reduction assay was carried out using 2,3-bis(2-methoxy-4-nitro-5-sulfophenyl)-5[(phenylamino)carbonyl]-2H-tetrazolium hydroxide (XTT; Sigma). XTT was dissolved at $0.5 \mathrm{~g} \mathrm{l}^{-1}$ in Ringer's lactate, sterilized through a $0.22 \mu \mathrm{m}$ filter, stored at $-70{ }^{\circ} \mathrm{C}$ and maintained at $4{ }^{\circ} \mathrm{C}$ when in use. Before use, $100 \mathrm{mM}$ menadione in acetone was added to a final concentration of $10 \mu \mathrm{M}$ (Ramage et al., 2001). At the same predetermined time points, another $100 \mu \mathrm{l}$ sample was removed from each solution into a 96-well flat-bottomed microtitre plate and a $100 \mu \mathrm{l}$ aliquot of XTT/menadione solution was added to each well. The plate was incubated in the dark for up to $2 \mathrm{~h}$ at $35{ }^{\circ} \mathrm{C}$. After this, the absorbance of the XTT reduction product, formazan, was read at $492 \mathrm{~nm}$ for each well using a plate reader (Lewis \& Kontoyiannis, 2005). All experiments were conducted in triplicate.

A standard correlation between viable cell counts from colony counting and optical density values from the XTT reduction assay was used to transform XTT reduction results into cell counts and to compare them. The following criteria were used for both methods to interpret time-kill results: synergy, $a \geqslant 2 \log _{10}$ decrease in c.f.u. $\mathrm{ml}^{-1}$ compared with the most active constituent; antagonism, $a \geqslant 2 \log _{10}$ increase in c.f.u. $\mathrm{ml}^{-1}$ compared with the least active agent; additivity, a $<2$ but $>1 \log _{10}$ decrease in c.f.u. $\mathrm{ml}^{-1}$ compared with the most active agent; and indifference, a $<2$ but $>1 \log _{10}$ increase in c.f.u. $\mathrm{ml}^{-1}$ compared with the least active agent (Mukherjee et al., 2005).

\section{RESULTS AND DISCUSSION}

\section{Susceptibility and interaction of drugs}

The MICs of FLC, ITC and VRC in each test, based on an $80 \%$ reduction in growth, for C. parapsilosis ATCC 22019 were $4,0.125$ and $0.063 \mu \mathrm{g} \mathrm{ml}^{-1}$, respectively, which are within the reference ranges. Table 1 summarizes the in vitro susceptibility of the drugs alone and in combination against $C$. albicans. The susceptibility of experimental isolates agreed well among the three azoles, indicating the existence of cross-resistance. Generally, for cross-resistant strains, the FICI ranged from 0.001 to 0.018 (much smaller than 0.5 ) and the percentages of synergistic interactions ranged from 691 to $2256 \%$ (far beyond $100 \%$ ), suggesting 
Table 1. Susceptibility of drugs alone and in combination against clinically resistant C. albicans

The MIC and FICI values are shown as the median of three independent experiments. R, Resistant; S, susceptible; INT, interpretation; SYN, synergism; ANT, antagonism; NI, no interaction; $n$, number of interactions.

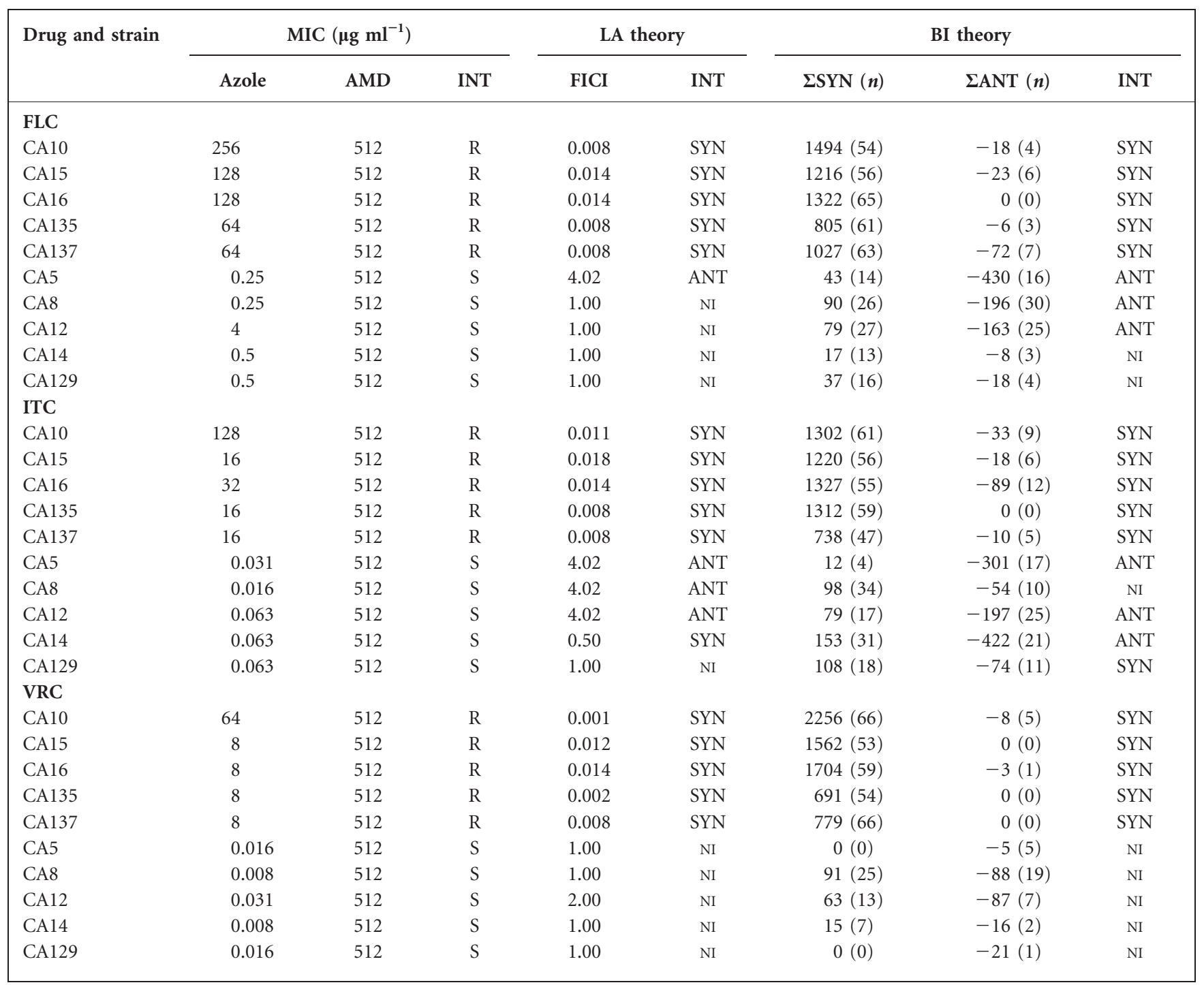

a very strong enhanced antifungal effect of azoles by AMD, whilst there was predominantly no synergistic effect observed for the tested susceptible C. albicans isolates. To obtain a visual characteristic of the drug combinations, a 3D surface plot of FLC combined with AMD against strain CA10 was prepared (Fig. 1).

In vitro interaction of two or more agents is dependent on the methodology used to generate the data, as well as on the way the results are analysed, resulting in variable as well as controversial conclusions (Meletiadis et al., 2003). Compared with the FICI, the Prichard model not only allows an objective statistical criterion, but also fits all the experimental concentrations to construct a 3D graph to visualize the nature and intensity of drug combinations without arbitrarily choosing an end point (Te Dorsthorst et al., 2002). Compared with the fully parametric and semi-parametric response surface approaches (Afeltra et al., 2004), the Prichard model is not dependent on the data analysis program and the sigmoid dose-response, and thus does not fail to fit the data. Last but most importantly, the Prichard model had an excellent reproducibility for the 77 combinations calculated for each strain. Current methods based on the LA and BI models are associated with implicit assumptions about the interacting system (Tam et al., 2004). Considering all of the above, we strongly advise the use of the Prichard model to assess the interaction between two drugs for its peerless advantages.

\section{Time-kill curves of drug combinations}

The optical density values from the XTT reduction assay were transformed into colony counts using the standard 


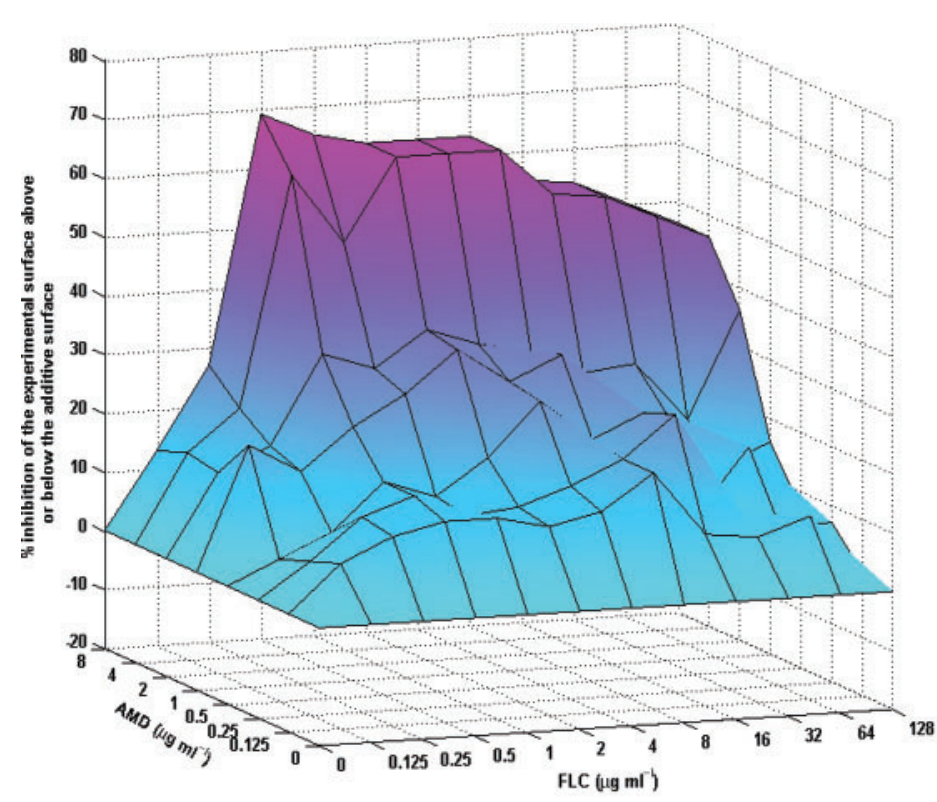

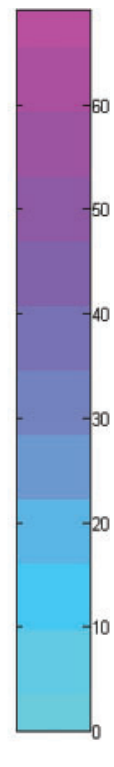

Fig. 1. Three-dimensional plot of the synergy of FLC in combination with AMD against strain CA10 using the MATLAB program. The mean $\triangle \mathrm{E}$ values obtained for three separate experiments were depicted on the $z$-axis to form the graph. Peaks above the 0 plane indicate synergistic combination. curve in Fig. 2. The results of AMD combined with the azoles from the time-kill study are shown in Table 2. Synergistic interactions from chequerboard testing were confirmed by time-kill curves, although we had to convert the colony counting data from FLC with AMD and ITC with AMD into integers. Representative plots of $\log _{10}$ c.f.u. $\mathrm{ml}^{-1}$ against time for the XTT reduction assay are shown in Fig. 3. AMD alone had a very weak antifungal effect at $4 \mu \mathrm{g} \mathrm{ml} \mathrm{m}^{-1}$ at $48 \mathrm{~h}$, but the fungistatic activity of azoles against the resistant strain (CA10) was dramatically enhanced by the addition of AMD. Given the initial inoculum of $10^{5}$ c.f.u. $\mathrm{ml}^{-1}$, the combination yielded, respectively, a mean decrease of 2.02, 2.04 and $2.08 \log _{10}$ c.f.u. $\mathrm{ml}^{-1}$ for FLC, ITC and VRC after incubation for $48 \mathrm{~h}$. There were no obvious differences in features of drug action among the three combinations.

Time-kill curves provided growth kinetic information over time and gave a more detailed picture of the effect of drug combinations on cell viability (Mukherjee et al., 2005). XTT is reduced by mitochondrial dehydrogenases of viable

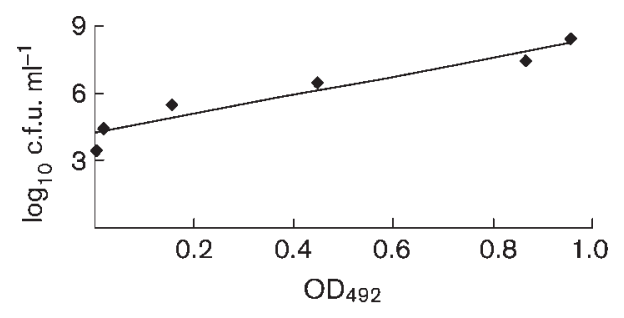

Fig. 2. Relationship between colony counts and results of the XTT reduction assay to measure the growth of $C$. albicans without drugs after $48 \mathrm{~h}$. Results are expressed as $y=4.261 x+4.2087$, with correlation coefficient $r=0.9622$. fungi into a water-soluble formazan product, which is measured by the absorbance at $492 \mathrm{~nm}$ (Kuhn et al., 2002). Previous work showed an actual relationship between Candida cell number and XTT formazan signal (Kuhn et al., 2003), so we carried out time-kill experiments using the XTT reduction assay and compared it with colony counting. Without the influence of filament formation, time-kill curves obtained using the XTT assay were as reliable as colony counting. The XTT assay remains a valuable tool for examining the growth and metabolism of yeast, whether in planktonic or biofilm form (Kuhn et al., 2003), and may become a substitute for colony counting for its time-saving advantage.

\section{Potential mechanisms of AMD that augment the antifungal effects of azoles}

The action of azoles not only involves impeding ergosterol biosynthesis through the competitive inhibition of $14 \alpha$ demethylase, which is a product of the ERG11 gene, but

Table 2. Decrease in c.f.u. $\mathrm{ml}^{-1}$ of strain CA10 using AMD combined with azoles at $48 \mathrm{~h}$

The combined decrease in c.f.u. $\mathrm{ml}^{-1}$ was compared with that of the corresponding azoles, and the triplicate results of experiments are reported as mean $\pm \mathrm{SD}$. INT, Interpretation; SYN, synergism.

\begin{tabular}{|lccccc|}
\hline \multirow{2}{*}{$\begin{array}{l}\text { Drug } \\
\text { combination }\end{array}$} & \multicolumn{2}{c}{ Colony count } & & \multicolumn{2}{c|}{ XTT reduction assay } \\
\cline { 2 - 3 } \cline { 6 - 6 } & Mean \pm SD & INT & & Mean \pm SD & INT \\
\hline FLC + AMD & $1.99 \pm 0.02$ & SYN & & $2.02 \pm 0.03$ & SYN \\
ITR + AMD & $1.99 \pm 0.02$ & SYN & & $2.04 \pm 0.03$ & SYN \\
VRC + AMD & $2.02 \pm 0.02$ & SYN & & $2.08 \pm 0.03$ & SYN \\
\hline
\end{tabular}



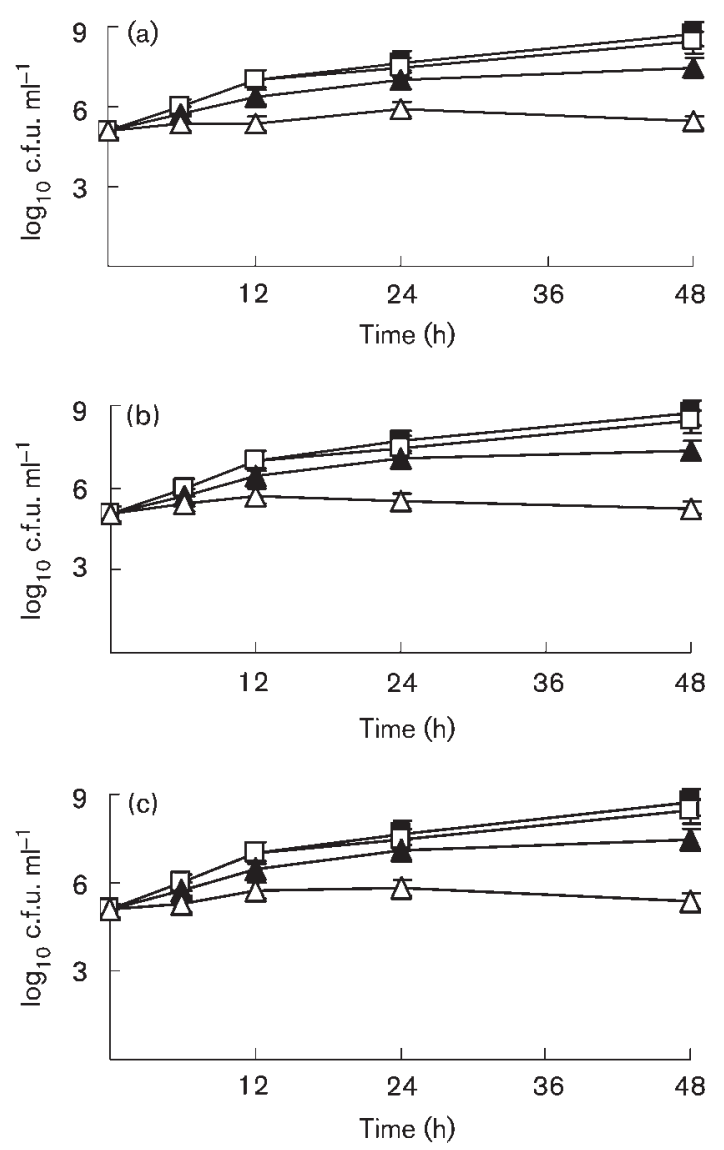

Fig. 3. Time-kill curves for strain CA10 obtained by XTT assay using $8 \mu \mathrm{g} \mathrm{ml}^{-1}$ for FLC (a) and $0.5 \mu \mathrm{g} \mathrm{ml}^{-1}$ for ITC (b) and VRC (c) combined with AMD at $4 \mu \mathrm{g} \mathrm{ml}^{-1}$. $\mathbf{\square}$, Growth control; $\square$, AMD alone; $\boldsymbol{\Delta}$, azole alone; $\triangle$, azole combined with AMD.

also leads to accumulation of 14-methylated sterol, which can cause disruption of fungal membranes (Morschhäuser, 2002; Ribeiro \& Paula, 2007). Our investigation illustrated that AMD used in combination with azoles acted synergistically against resistant $C$. albicans strains, whilst there was almost no synergistic activity seen against susceptible strains. Considering the effect of AMD in inhibiting L-type $\mathrm{Ca}^{2+}$ channels and $\mathrm{Na}^{+} / \mathrm{K}^{+}$channels when treating atrial fibrillation in patients with left ventricular dysfunction and heart failure, we propose that the most likely mechanism of the synergistic effect would be a consequence of disrupted $\mathrm{Ca}^{2+}$ homeostasis as reported by Gupta et al. (2003). Other possible mechanisms whereby AMD could reduce the overexpression of genes encoding efflux pumps or azole target enzymes are still under study.

Although AMD can cause serious adverse effects, including anaemia (Nicolay et al., 2007), corneal microdeposits, optic neuropathy/neuritis, blue-grey skin discoloration, photosensitivity, hypothyroidism, hyperthyroidism, pulmonary toxicity, peripheral neuropathy and hepatotoxicity (Vassallo \& Trohman, 2007), as one of the first-line agents of atrial fibrillation treatment, it is widely used in clinical situations. In addition, this study provides a new clue to antifungal mechanisms by considering the known actions of AMD, and suggests a way of treating resistant C. albicans infection through a drug combination approach.

In conclusion, our results highlight the promising role of AMD used in combination with azoles to treat serious resistant C. albicans infection. Prospective studies in appropriate animal models are required to develop therapeutic strategies for these combinations, and further studies in other types of fungi and with a larger number of strains are needed.

\section{ACKNOWLEDGEMENTS}

This work was supported by a grant (2005GG4402036) from the Science and Technology of Shandong Province, PR China.

\section{REFERENCES}

Afeltra, J., Vitale, R. G., Mouton, J. W. \& Verweij, P. E. (2004). Potent synergistic in vitro interaction between nonantimicrobial membraneactive compounds and itraconazole against clinical isolates of Aspergillus fumigatus resistant to itraconazole. Antimicrob Agents Chemother 48, 1335-1343.

Andes, D., Forrest, A., Lepak, A., Nett, J., Marchillo, K. \& Lincoln, L. (2006). Impact of antimicrobial dosing regimen on evolution of drug resistance in vivo: fluconazole and Candida albicans. Antimicrob Agents Chemother 50, 2374-2383.

Barchiesi, F., Di Francesco, L. F., Compagnucci, P., Arzeni, D., Giacometti, A. \& Scalise, G. (1998). In-vitro interaction of terbinafine with amphotericin B, fluconazole and itraconazole against clinical isolates of Candida albicans. J Antimicrob Chemother 41, 59-65.

Baumgartner, C., Freydiere, A. M. \& Gille, Y. (1996). Direct identification and recognition of yeast species from clinical material by using albicans ID and CHROMagar Candida plates. J Clin Microbiol 34, 454-456.

CLSI (2002). Reference Method for Broth Dilution Antifungal Susceptibility Testing of Yeasts. Approved standard M27-A2. Wayne, PA: Clinical Laboratory Standards Institute.

Courchesne, W. E. (2002). Characterization of a novel, broad-based fungicidal activity for the antiarrhythmic drug amiodarone. J Pharmacol Exp Ther 300, 195-199.

Cruz, M. C., Goldstein, A. L., Blankenship, J. R., Del Poeta, M., Davis, D., Cardenas, M. E., Perfect, J. R., McCusker, J. H. \& Heitman, J. (2002). Calcineurin is essential for survival during membrane stress in Candida albicans. EMBO J 21, 546-559.

Espinel-Ingroff, A., Barchiesi, F., Cuenca-Estrella, M., Pfaller, M. A., Rinaldi, M., Rodriguez-Tudela, J. L. \& Verweij, P. E. (2005a). International and multicenter comparison of EUCAST and CLSI M27-A2 broth microdilution methods for testing susceptibilities of Candida spp. to fluconazole, itraconazole, posaconazole, and voriconazole. J Clin Microbiol 43, 3884-3889.

Espinel-Ingroff, A., Barchiesi, F., Cuenca-Estrella, M., Fothergill, A., Pfaller, M. A., Rinaldi, M., Rodriguez-Tudela, J. L. \& Verweij, P. E. (2005b). Comparison of visual 24-hour and spectrophotometric 48hour MICs to CLSI reference microdilution MICs of fluconazole, itraconazole, posaconazole, and voriconazole for Candida spp.: a collaborative study. J Clin Microbiol 43, 4535-4540. 
Filler, S. G. \& Sheppard, D. C. (2006). Fungal invasion of normally non-phagocytic host cells. PLoS Pathog 2, e129.

Fricker-Hidalgo, H., Vandapel, O., Duchesne, M. A., Mazoyer, M. A., Monget, D., Lardy, B., Lebeau, B., Freney, J., Ambroise-Thomas, P. \& Grillot, R. (1996). Comparison of the new API Candida system to the ID 32C system for identification of clinically important yeast species. J Clin Microbiol 34, 1846-1848.

Ghannoum, M. A., Fu, Y., Ibrahim, A. S., Mortara, L. A., Shafiq, M. C., Edwards, J. E., Jr \& Criddle, R. S. (1995). In vitro determination of optimal antifungal combinations against Cryptococcus neoformans and Candida albicans. Antimicrob Agents Chemother 39, 2459-2465.

Girmenia, C., Venditti, M. \& Martino, P. (2003). Fluconazole in combination with flucytosine in the treatment of fluconazole-resistant Candida infections. Diagn Microbiol Infect Dis 46, 227-231.

Gupta, S. S., Ton, V. K., Beaudry, V., Rulli, S., Cunningham, K. \& Rao, R. (2003). Antifungal activity of amiodarone is mediated by disruption of calcium homeostasis. J Biol Chem 278, 28831-28839.

Klepser, M. E., Ernst, E. J., Lewis, R. E., Ernst, M. E. \& Pfaller, M. A. (1998). Influence of test conditions on antifungal time-kill curve results: proposal for standardized methods. Antimicrob Agents Chemother 42, 1207-1212.

Kuhn, D. M., Chandra, J., Mukherjee, P. K. \& Ghannoum, M. A. (2002). Comparison of biofilms formed by Candida albicans and Candida parapsilosis on bioprosthetic surfaces. Infect Immun 70, 878-888.

Kuhn, D. M., Balkis, M., Chandra, J., Mukherjee, P. K. \& Ghannoum, M. A. (2003). Uses and limitations of the XTT assay in studies of Candida growth and metabolism. J Clin Microbiol 41, 506-508.

Lewis, R. E. \& Kontoyiannis, D. P. (2005). Micafungin in combination with voriconazole in Aspergillus species: a pharmacodynamic approach for detection of combined antifungal activity in vitro. J Antimicrob Chemother 56, 887-892.

Meletiadis, J., Mouton, J. W., Meis, J. F. \& Verweij, P. E. (2003). In vitro drug interaction modeling of combinations of azoles with terbinafine against clinical Scedosporium prolificans isolates. Antimicrob Agents Chemother 47, 106-117.

Morschhäuser, J. (2002). The genetic basis of fluconazole resistance development in Candida albicans. Biochim Biophys Acta 1587, 240-248.

Mukherjee, P. K., Sheehan, D. J., Hitchcock, C. A. \& Ghannoum, M. A. (2005). Combination treatment of invasive fungal infections. Clin Microbiol Rev 18, 163-194.
Nicolay, J. P., Bentzen, P. J., Ghashghaeinia, M., Wieder, T. \& Lang, F. (2007). Stimulation of erythrocyte cell membrane scrambling by amiodarone. Cell Physiol Biochem 20, 1043-1050.

Odds, F. C. (2003). Synergy, antagonism, and what the chequerboard puts between them. J Antimicrob Chemother 52, 1.

Pfaller, M. A., Houston, A. \& Coffmann, S. (1996). Application of CHROMagar Candida for rapid screening of clinical specimens for Candida albicans, Candida tropicalis, Candida krusei and Candida (Torulopsis) glabrata. J Clin Microbiol 34, 58-61.

Pfaller, M. A., Boyken, L., Hollis, R. J., Messer, S. A., Tendolkar, S. \& Diekema, D. J. (2005). In vitro susceptibilities of clinical isolates of Candida species, Cryptococcus neoformans, and Aspergillus species to itraconazole: global survey of 9,359 isolates tested by Clinical and Laboratory Standards Institute broth microdilution methods. J Clin Microbiol 43, 3807-3810.

Quan, H., Cao, Y. Y., Xu, Z., Zhao, J. X., Gao, P. H., Qin, X. F. \& Jiang, Y. Y. (2006). Potent in vitro synergism of fluconazole and berberine chloride against clinical isolates of Candida albicans resistant to fluconazole. Antimicrob Agents Chemother 50, 1096-1099.

Ramage, G., Vande Walle, K., Wickes, B. L. \& López-Ribot, J. L. (2001). Standardized method for in vitro antifungal susceptibility testing of Candida albicans biofilms. Antimicrob Agents Chemother 45, 2475-2479.

Ribeiro, M. A. \& Paula, C. R. (2007). Up-regulation of ERG11 gene among fluconazole-resistant Candida albicans generated in vitro: is there any clinical implication? Diagn Microbiol Infect Dis 57, 71-75.

Tam, V. H., Schilling, A. N., Lewis, R. E., Melnick, D. A. \& Boucher, A. N. (2004). Novel approach to characterization of combined pharmacodynamic effects of antimicrobial agents. Antimicrob Agents Chemother 48, 4315-4321.

Te Dorsthorst, D. T., Verweij, P. E., Meis, J. F., Punt, N. C. \& Mouton, J. W. (2002). Comparison of fractional inhibitory concentration index with response surface modeling for characterization of in vitro interaction of antifungals against itraconazole-susceptible and -resistant Aspergillus fumigatus isolates. Antimicrob Agents Chemother 46, 702-707.

Te Dorsthorst, D. T., Verweij, P. E., Meis, J. F., Punt, N. C. \& Mouton, J. W. (2004). In vitro interactions between amphotericin B, itraconazole, and flucytosine against 21 clinical Aspergillus isolates determined by two drug interaction models. Antimicrob Agents Chemother 48, 2007-2013.

Vassallo, P. \& Trohman, R. G. (2007). Prescribing amiodarone: an evidence-based review of clinical indications. JAMA 298, 1312-1322. 